\title{
Kerr-Schild spacetimes with (A)dS background
}

\author{
Tomáš Málek ${ }^{1}$, Vojtěch Pravda ${ }^{2}$ \\ 1 Institute of Theoretical Physics, Faculty of Mathematics and Physics, \\ Charles University, V Holešovičkách 2, 18000 Prague 8, Czech Republic \\ E-mail: malek@math.cas.cz \\ 2 Institute of Mathematics, Academy of Sciences of the Czech Republic, \\ Žitná 25, 11567 Prague 1, Czech Republic \\ E-mail: pravda@math.cas.cz
}

\begin{abstract}
General properties of Kerr-Schild spacetimes with (A)dS background in arbitrary dimension $n>3$ are studied. It is shown that the geodetic Kerr-Schild vector $\boldsymbol{k}$ is a multiple WAND of the spacetime. Einstein Kerr-Schild spacetimes with non-expanding $\boldsymbol{k}$ are shown to be of Weyl type $\mathrm{N}$, while the expanding spacetimes are of type II or D.

It is shown that this class of spacetimes obeys the optical constraint. This allows us to solve Sachs equation, determine $r$-dependence of boost weight zero components of the Weyl tensor and discuss curvature singularities.

PACS numbers: 04.50.-h, 04.20.Jb, 04.20.Cv
\end{abstract}

\section{Introduction}

Kerr-Schild (KS) class of spacetimes [1], i.e. metrics of the form

$$
g_{a b}=\eta_{a b}-2 \mathcal{H} k_{a} k_{b},
$$

with $\mathcal{H}$ being a scalar function and $\boldsymbol{k}$ being a null vector with respect to the background flat metric $\eta_{a b}$ and full metric $g_{a b}$, play an important role in the study of exact solutions of the vacuum Einstein equations in four and higher dimensions. The exceptional advantage of this ansatz is that it makes analytic calculations tractable and allows analysis of such spacetimes in full generality while at the same time it contains exact solutions of high interest, such as Kerr black holes and higher dimensional MyersPerry black holes [2] and type N pp-waves [3, 4]. General properties of such metrics in arbitrary dimension were studied in [4].

Rotating black holes with de Sitter and anti-de Sitter backgrounds discovered in four, five and higher dimensions in [5], [6] and [7], respectively, can be cast to the generalized Kerr-Schild (GKS) form $\ddagger$

$$
g_{a b}=\bar{g}_{a b}-2 \mathcal{H} k_{a} k_{b},
$$

with $\boldsymbol{k}$ again being null vector with respect to background de Sitter or anti-de Sitter metric $\bar{g}_{a b}$ and full metric $g_{a b}$.

In this paper we analyze properties of metrics (2) in dimension $n>3$ and generalize the main results of [4] from the Ricci flat case to the case of Einstein

$\ddagger$ See e.g. $[8,7]$ for the discussion of this class of metrics in higher dimensions. 
spacetimes. Hereafter we thus assume that $\bar{g}_{a b}=\Omega \eta_{a b}$ is $n$-dimensional (A)dS metric with cosmological constant $\Lambda$, with Minkowski metric $\eta_{a b}$ being in the canonical form $-\mathrm{d} t^{2}+\mathrm{d} x_{1}^{2}+\ldots+\mathrm{d} x_{n-1}{ }^{2}$.

In section 2 it is shown that under quite general conditions, including the case of Einstein spacetimes, Einstein equations imply that the KS vector field $\boldsymbol{k}$ is geodetic. In section 3 curvature tensors and Einstein equations for the metric (2) are studied in the case of geodetic $\boldsymbol{k}$. It is also shown that $\boldsymbol{k}$ is necessarily a multiple WAND.

In the rest of the paper we focus on Einstein GKS spacetimes. In section 4 we point out that Brinkmann warp product preserves GKS form. In section 5 it is shown that for the non-expanding $\boldsymbol{k}$ Einstein GKS spacetimes belong to type N Kundt class and explicit examples of such metrics are obtained using the Brinkmann warp product. In section 6 we study the case with expanding $\boldsymbol{k}$. It is shown that these spacetimes obey the "optical constraint" [4]. This allows us to determine $r$-dependence $\S$ of the optical matrix and boost weight zero components of the curvature tensors and analyze curvature singularities.

In section 7 we briefly discuss the main results. Appendix A contains frame components of Riemann and Weyl tensors in the case of geodetic KS vector $\boldsymbol{k}$. In the Appendix B we compare the $r$-dependence of the optical matrix in a parallelly propagated frame for general Einstein GKS metric and for the five-dimensional (A)dSKerr black hole.

\subsection{Preliminaries}

Throughout the paper we use standard notation of higher dimensional NP formalism $[9,10]$ (see also [11]). For completeness, let us briefly summarize the notation and list several useful relations.

We will work in a real frame $\boldsymbol{n} \equiv \boldsymbol{m}^{(0)}, \boldsymbol{\ell} \equiv \boldsymbol{m}^{(1)}, \boldsymbol{m}^{(i)}$ consisting of two null vectors $\boldsymbol{\ell}, \boldsymbol{n}$ and $n-2$ orthonormal spacelike vectors $\boldsymbol{m}^{(i)}$ obeying

$\ell^{a} \ell_{a}=n^{a} n_{a}=\ell^{a} m_{a}^{(i)}=n^{a} m_{a}^{(i)}=0, \quad \ell^{a} n_{a}=1, \quad m^{(i) a} m_{a}^{(j)}=\delta_{i j}$,

with indices $i, j, \ldots$ going from 2 to $n-1$ and $a, b, \ldots$ from 0 to $n-1$. Then the full metric takes the form

$$
g_{a b}=2 n_{(a} \ell_{b)}+\delta_{i j} m_{a}^{(i)} m_{b}^{(j)} .
$$

Throughout the paper we conveniently identify the KS vector $\boldsymbol{k}$ with the null frame vector $\ell$.

Ricci rotation coefficients $L_{a b}, N_{a b}$ and $\stackrel{i}{M} b c$ are defined as the frame components of covariant derivatives

$\ell_{a ; b}=L_{c d} m_{a}^{(c)} m_{b}^{(d)}, \quad n_{a ; b}=N_{c d} m_{a}^{(c)} m_{b}^{(d)}, \quad m_{a ; b}^{(i)}=\stackrel{i}{M}_{c d} m_{a}^{(c)} m_{b}^{(d)}$.

In the case of geodetic and affinely parametrized vector $\boldsymbol{\ell}$ the following definitions $[9,10]$ are useful

$$
\begin{aligned}
& S_{i j} \equiv L_{(i j)}=\sigma_{i j}+\theta \delta_{i j}, \quad A_{i j} \equiv L_{[i j]}, \\
& \theta \equiv \frac{1}{n-2} S_{i i}, \quad \sigma^{2} \equiv \sigma_{i j} \sigma_{i j}, \quad \omega^{2} \equiv A_{i j} A_{i j},
\end{aligned}
$$

where $S_{i j}, \sigma_{i j}$ and $A_{i j}$ are the expansion, shear and twist matrices, respectively, and $\theta, \sigma$ and $\omega$ are the corresponding scalars.

$\S$ With $r$ being the affine parameter along KS congruence $\boldsymbol{k}$. 
Directional derivatives along the frame vectors are denoted as

$$
\mathrm{D} \equiv \ell^{a} \nabla_{a}, \quad \Delta \equiv n^{a} \nabla_{a}, \quad \delta_{i} \equiv m_{(i)}^{a} \nabla_{a} .
$$

Finally, the conformal factor $\Omega$ in the background de Sitter and anti-de Sitter metric $\bar{g}_{a b}=\Omega \eta_{a b}$ is

$$
\begin{aligned}
& \Omega=\Omega^{+}=\frac{\ell_{\Lambda}^{2}}{t^{2}}=\frac{(n-2)(n-1)}{2 \Lambda t^{2}}, \\
& \Omega=\Omega^{-}=\frac{a^{2}}{x_{1}{ }^{2}}=-\frac{(n-2)(n-1)}{2 \Lambda x_{1}{ }^{2}},
\end{aligned}
$$

respectively, while Minkowski limit $\Lambda=0$ can be obtained by setting $\Omega=1$. Note also that $\Omega$ satisfies

$$
\frac{\Omega_{, a b}}{\Omega}=\frac{3}{2} \frac{\Omega_{, a} \Omega_{, b}}{\Omega^{2}}, \quad-\frac{1}{4} \frac{\Omega_{, a} \Omega_{, b}}{\Omega^{2}} \bar{g}^{a b}=\frac{2}{(n-2)(n-1)} \Lambda .
$$

When $\boldsymbol{k}$ is geodetic and affinely parametrized, the following identities are also useful

$$
k_{; a}^{a}=L_{i i}, \quad k_{a ; b} k^{a ; b}=L_{i j} L_{i j}, \quad k_{a ; b} k^{b ; a}=L_{i j} L_{j i} .
$$

\section{General KS vector field}

The main point of this section is to show that if energy-momentum tensor obeys $T_{a b} k^{a} k^{b}=0$ then Einstein equations imply that KS vector field is geodetic. This fact is then used in the following sections.

Inverse metric to (2) has the form

$$
g^{a b}=\bar{g}^{a b}+2 \mathcal{H} k^{a} k^{b},
$$

where $\bar{g}^{a b}=\Omega^{-1} \eta^{a b}$. Christoffel symbols read

$\Gamma_{b c}^{a}=-\left(\mathcal{H} k^{a} k_{b}\right)_{, c}-\left(\mathcal{H} k^{a} k_{c}\right)_{, b}+g^{a s}\left(\mathcal{H} k_{b} k_{c}\right)_{, s}+\frac{1}{2} \frac{\Omega_{, c}}{\Omega} \delta_{b}^{a}+\frac{1}{2} \frac{\Omega_{, b}}{\Omega} \delta_{c}^{a}-\frac{1}{2} \frac{\Omega_{, s}}{\Omega} g^{a s} \bar{g}_{b c}$.

When studying constraints following from the Einstein equations, it is natural to start with the highest boost weight component of the Ricci tensor $R_{00}=R_{a b} k^{a} k^{b}$ - since $k$ is present in $\Gamma_{b c}^{a}$, many terms in this contraction vanish. Though such calculation is still quite involved it leads to a remarkably simple result

$$
R_{00}=2 \mathcal{H} k_{c ; a} k^{a} k_{; b}^{c} k^{b}-\frac{1}{2}(n-2)\left(\frac{\Omega_{, a b}}{\Omega}-\frac{3}{2} \frac{\Omega_{, a} \Omega_{, b}}{\Omega^{2}}\right) k^{a} k^{b}
$$

for general form of $\Omega$. Therefore for (A)dS background from (10)

$$
R_{00}=2 \mathcal{H} k_{c ; a} k^{a} k_{; b}^{c} k^{b} .
$$

From the Einstein equations it now follows

Proposition 1 The null vector $k^{a}$ in the generalized Kerr-Schild metric (2) is geodetic if and only if the component of the energy-momentum tensor $T_{00}=T_{a b} k^{a} k^{b}$ vanishes.

Proposition 1 implies that vector $\boldsymbol{k}$ is geodetic for Einstein GKS spacetimes. In fact geodeticity of $\boldsymbol{k}$ also holds for spacetimes with aligned matter fields such as aligned Maxwell field $\left(F_{a b} k^{a} \propto k_{b}\right)$ or aligned pure radiation $\left(T_{a b} \propto k_{a} k_{b}\right)$. Thus starting from section 3 we consider $\boldsymbol{k}$ being geodetic and affinely parametrized. This leads to a considerable simplification of the necessary calculations. 


\subsection{KS congruence in the background spacetime}

Here we point out that geodeticity and optical properties of the KS congruence in the background (A)dS spacetime and in the full GKS spacetime coincide.

Note that Christoffel symbols and curvature tensor components of the background (A)dS spacetime can be obtained from the corresponding quantities in the full GKS spacetime by simply setting $\mathcal{H}$ to zero. Using (13) it is straightforward to see that

$$
\begin{aligned}
& k_{a ; b} k^{b}=k_{a, b} k^{b}=k_{a ; b} k^{b}, \\
& k_{; b}^{a} k^{b}=k_{, b}^{a} k^{b}+\frac{\Omega_{, b}}{\Omega} k^{a} k^{b}=k_{; b}^{a} k^{b},
\end{aligned}
$$

where $k_{a ; b}$ denotes a covariant derivative with respect to the background (A)dS metric $\bar{g}_{a b}$. Thus $\boldsymbol{k}$ is geodetic in the full GKS metric iff it is geodetic in the (A)dS background $\bar{g}_{a b}$.

Following [4] we can introduce a null frame in the background $\bar{g}_{a b}$ by replacing $\boldsymbol{n}$ by $\tilde{\boldsymbol{n}}$ and keeping remaining frame vectors unchanged

$$
\tilde{n}_{a}=n_{a}+\mathcal{H} k_{a},
$$

which guarantees

$$
\bar{g}_{a b}=2 k_{(a} \tilde{n}_{b)}+\delta_{i j} m_{a}^{(i)} m_{b}^{(j)}
$$

and allows us to compare the optical matrices $L_{i j}$ and $\tilde{L}_{i j}$ in the full spacetime and

in the background, respectively. Note that for $\boldsymbol{k}$ geodetic, $L_{i j}$ does not depend on our particular choice (17) since in such case $L_{i j}$ is invariant under null rotations with $\boldsymbol{k}$ fixed [10].

Using (13) it follows

$$
L_{i j} \equiv k_{a ; b} m^{(i) a} m^{(j) b}=k_{a ; b} m^{(i) a} m^{(j) b} \equiv \tilde{L}_{i j}
$$

and therefore the optical matrices of the congruence $\boldsymbol{k}$ in the full GKS spacetime and in the (A)dS background are equal.

\section{Curvature tensors for geodetic KS vector field}

As discussed in section 2, for Einstein GKS spacetimes KS vector $\boldsymbol{k}$ is always geodetic and therefore from now on we assume geodeticity of $\boldsymbol{k}$. Then we arrive at convenient expressions used in the following calculations

$$
\begin{aligned}
\Gamma_{b c}^{a} k^{b} & =-\mathrm{DH} k^{a} k_{c}+\frac{1}{2} \frac{\Omega_{, c}}{\Omega} k^{a}+\frac{1}{2} \frac{\Omega_{, b}}{\Omega} k^{b} \delta_{c}^{a}-\frac{1}{2} \frac{\Omega_{, b}}{\Omega} \bar{g}^{a b} k_{c}, \\
\Gamma_{b c}^{a} k_{a} & =\mathrm{D} \mathcal{H} k_{b} k_{c}+\frac{1}{2} \frac{\Omega_{, c}}{\Omega} k_{b}+\frac{1}{2} \frac{\Omega_{, b}}{\Omega} k_{c}-\frac{1}{2} \frac{\Omega, a}{\Omega} k^{a} \bar{g}_{b c} .
\end{aligned}
$$

\subsection{Ricci tensor}

Ricci tensor of the GKS metric can be expressed as

$$
\begin{aligned}
R_{a b}= & \left(\mathcal{H} k_{a} k_{b}\right)_{; c d} g^{c d}-\left(\mathcal{H} k^{s} k_{a}\right)_{; b s}-\left(\mathcal{H} k^{s} k_{b}\right)_{; a s}+\frac{2 \Lambda}{n-2} \bar{g}_{a b} \\
& -2 \mathcal{H}\left(\mathrm{D}^{2} \mathcal{H}+L_{i i} \mathrm{DH}+2 \mathcal{H} \omega^{2}\right) k_{a} k_{b},
\end{aligned}
$$


which for $\Lambda=0$ reduces to the result of [4]. From (22) it follows that $\boldsymbol{k}$ is an eigenvector of the Ricci tensor

$$
R_{a b} k^{b}=-\left[D^{2} \mathcal{H}+(n-2) \theta D \mathcal{H}+2 \mathcal{H} \omega^{2}-\frac{2 \Lambda}{n-2}\right] k_{a}
$$

and thus boost weight 1 frame components $R_{0 i}$ of the Ricci tensor vanish along with $R_{00}$. The non-vanishing frame components of the Ricci tensor read

$$
\begin{aligned}
& R_{01}=-\mathrm{D}^{2} \mathcal{H}-(n-2) \theta \mathrm{D} \mathcal{H}-2 \mathcal{H} \omega^{2}+\frac{2 \Lambda}{n-2} \\
& R_{i j}=2 \mathcal{H} L_{i k} L_{j k}-2(\mathrm{D} \mathcal{H}+(n-2) \theta \mathcal{H}) S_{i j}+\frac{2 \Lambda}{n-2} \delta_{i j} \\
& R_{1 i}=-\delta_{i}(\mathrm{D} \mathcal{H})+2 L_{[i 1]} \mathrm{D} \mathcal{H}+2 L_{i j} \delta_{j} \mathcal{H}-S_{j j} \delta_{i} \mathcal{H} \\
& \quad+2 \mathcal{H}\left(\delta_{j} A_{i j}+A_{i j} \stackrel{M}{k k}_{k k}-A_{j k} \stackrel{i}{j k}_{j k}-L_{1 i} S_{j j}+3 L_{i j} L_{[1 j]}+L_{j i} L_{(1 j)}\right) \\
& R_{11}=\delta_{i}\left(\delta_{i} \mathcal{H}\right)+\left(N_{i i}-2 \mathcal{H} S_{i i}\right) \mathrm{DH}+\left(4 L_{1 i}-2 L_{i 1}+\stackrel{i}{M_{j j}}\right) \delta_{i} \mathcal{H}-S_{i i} \Delta \mathcal{H}+\frac{4 \mathcal{H} \Lambda}{n-1} \\
& \quad+2 \mathcal{H}\left(2 \delta_{i} L_{[1 i]}+4 L_{1 i} L_{[1 i]}+L_{i 1} L_{i 1}-L_{11} S_{i i}+2 L_{[1 i]}^{i} M_{j j}-2 A_{i j} N_{i j}-2 \mathcal{H} \omega^{2}\right) .
\end{aligned}
$$

\subsection{Algebraic type of the Weyl tensor}

Components of the Weyl and Riemann tensor for the GKS metric with geodetic $\boldsymbol{k}$ are given in the Appendix A. In the previous section we have seen that positive boost weight frame components of the Ricci tensor identically vanish. It turns out that this is true for the Weyl tensor as well, i.e.

$$
C_{0 i 0 j}=0, \quad C_{010 i}=0, \quad C_{0 i j k}=0,
$$

and therefore

Proposition 2 Generalized Kerr-Schild spacetime (2) with a geodetic Kerr-Schild vector $\boldsymbol{k}$ is algebraically special with $\boldsymbol{k}$ being the multiple WAND.

KS spacetimes (2) with a geodetic Kerr-Schild vector $\boldsymbol{k}$ are therefore of Weyl type II or more special. Using also a result from [12] that spacetimes (not necessarily of the Kerr-Schild class) which are either static or stationary with non-vanishing "expansion" and "reflection symmetry" are compatible only with Weyl types G, I $i$, D or $\mathrm{O}$ we immediately arrive at

Corollary 3 Static generalized Kerr-Schild spacetimes (2) with a geodetic KerrSchild vector $\boldsymbol{k}$ are of type D or conformally flat.

Similar statement also holds for the stationary case. Note that the above proposition is not restricted to Einstein spaces - the only assumption we need is that $\boldsymbol{k}$ is geodetic, which is by proposition 1 equivalent to $T_{00}=T_{a b} k^{a} k^{b}=0$.

Note also that these results immediately imply that Kerr-de Sitter metrics in arbitrary dimension [7] are of type D, as shown previously in [13] by explicit calculation of the Weyl tensor. 


\subsection{Vacuum Einstein equations}

Since all previous results were derived without imposing Einstein field equations we now proceed with studying their implications for GKS spacetimes. From now on, we thus consider only Einstein spacetimes. Let us recall that in this case $\boldsymbol{k}$ is necessarily geodetic by proposition 1. Vacuum Einstein field equations (with cosmological constant) read

$$
R_{a b}=\frac{2}{n-2} \Lambda g_{a b}
$$

Note that the terms containing cosmological constant $\Lambda$ in the boost weight zero Ricci components $R_{01}$ (24) and $R_{i j}$ (25) cancel with the corresponding terms on the right hand side of the Einstein equations (29). The frame components of Einstein vacuum equations thus read

$$
\begin{aligned}
& \mathrm{D}^{2} \mathcal{H}+(n-2) \theta \mathrm{D} \mathcal{H}+2 \mathcal{H} \omega^{2}=0, \\
& 2 \mathcal{H} L_{i k} L_{j k}-2(\mathrm{D} \mathcal{H}+(n-2) \theta \mathcal{H}) S_{i j}=0, \\
& R_{1 i}=0, \quad R_{11}=0,
\end{aligned}
$$

where $R_{1 i}$ and $R_{11}$ are given by (26) and (27), respectively.

Following [4], we rewrite trace of (31) as

$$
\begin{aligned}
(n-2) \theta(\mathrm{D} \log \mathcal{H}) & =L_{i j} L_{i j}-(n-2)^{2} \theta^{2} \\
& =\sigma^{2}+\omega^{2}-(n-2)(n-3) \theta^{2} .
\end{aligned}
$$

Since $\mathcal{H}$ appears in (33) only for $\theta \neq 0$ it is natural to study non-expanding KS solutions $(\theta=0)$ and expanding KS solutions $(\theta \neq 0)$ separately. This will be done in sections 5 and 6 .

\section{Brinkmann warp product preserves GKS form}

In this section we point out that Brinkmann warp product (see $[14,15])$ preserves GKS form. This warp product is thus a convenient method for constructing $n$ dimensional Einstein GKS spacetimes from known $(n-1)$-dimensional GKS Ricci-flat or Einstein metrics. This applies e.g. to black strings constructed from Myers-Perry black holes with cosmological constant or to Einstein Kundt metrics constructed from Ricci-flat/Einstein Kundt metrics (see section 5).

It follows from [14] that starting with $(n-1)$-dimensional seed Einstein metric $\mathrm{d} \tilde{s}^{2}$, one can generate $n$-dimensional Einstein metric $\mathrm{d} s^{2}$

$$
\mathrm{d} s^{2}=\frac{1}{f(z)} \mathrm{d} z^{2}+f(z) \mathrm{d} \tilde{s}^{2},
$$

with

$$
f(z)=-\lambda z^{2}+2 d z+b, \quad \lambda=\frac{2 \Lambda}{(n-1)(n-2)},
$$

and with $b$ and $d$ being constant parameters. Necessary and sufficient condition for $\mathrm{d} s^{2}$ being Einstein spacetime is that

$$
\tilde{R}=(n-1)(n-2)\left(\lambda b+d^{2}\right),
$$

where $\tilde{R}$ is the Ricci scalar of the $(n-1)$-dimensional Einstein seed metric $\mathrm{d} \tilde{s}^{2}$. Note that for $R \neq 0$ only following combinations of signs of $\tilde{R}$ and $R$ are allowed: $(-,-)$, 
$(0,-),(+,-),(+,+)$ and that only the case $(-,-)$ is free from singularities at $f(z)=0$ (see [15]) .

It was also shown in [15] that Weyl type of $\mathrm{d} s^{2}$ is the same or more special than the type of $\mathrm{d} \tilde{s}^{2}$. In particular, if the seed metric $\mathrm{d} \tilde{s}^{2}$ is of type $\mathrm{N}$ then $\mathrm{d} s^{2}$ is of the type $\mathrm{N}$ as well.

Furthermore, if the seed metric $\mathrm{d} \tilde{s}^{2}$ is of the GKS form (2), then $\mathrm{d} s^{2}$ given by

$$
\frac{1}{f} \mathrm{~d} z^{2}+f \bar{g}_{a b} \mathrm{~d} x^{a} \mathrm{~d} x^{b}-2 f \mathcal{H} k_{a} k_{b} \mathrm{~d} x^{a} \mathrm{~d} x^{b} .
$$

is also of the GKS form since the new warped background metric $f^{-1} \mathrm{~d} z^{2}+f \bar{g}_{a b} \mathrm{~d} x^{a} \mathrm{~d} x^{b}$ is necessarily Einstein and conformally flat and therefore (A)dS or Minkowski.

For setting the warped background metric to the canonical form, one may use following coordinate transformations:

$$
\begin{array}{lll}
\operatorname{AdS}_{n-1} \Rightarrow \operatorname{AdS}_{n}: & x^{2}=\tilde{x}^{2}+\tilde{z}^{2}, & z=\frac{\sqrt{-\left(d^{2}+\lambda b\right)} \tilde{z}}{\lambda \tilde{x}}+\frac{d}{\lambda}, \\
\mathrm{dS}_{n-1} \Rightarrow \operatorname{AdS}_{n}: & t^{2}=\tilde{t}^{2}-\tilde{z}^{2}, & z=\frac{\sqrt{d^{2}+\lambda b} \tilde{t}}{-\lambda \tilde{z}}+\frac{d}{\lambda}, \\
\mathrm{dS}_{n-1} \Rightarrow \mathrm{dS}_{n}: & t^{2}=\tilde{t}^{2}-\tilde{z}^{2}, & z=\frac{\sqrt{d^{2}+\lambda b} \tilde{z}}{\lambda \tilde{t}}+\frac{d}{\lambda}, \\
\mathrm{M}_{n-1} \Rightarrow \operatorname{AdS}_{n}: & z=\frac{1}{\lambda \tilde{z}}+\frac{d}{\lambda} . &
\end{array}
$$

By an appropriate coordinate transformation one can set the warped metric (34) in a form conformal to a direct product [15]. Such form depends on the combination of signs of $\tilde{R}$ and $R$ and all such combinations are given in [15]. Here we list only cases relevant in this paper - i.e. cases with $R \neq 0$

$$
\begin{array}{lll}
\lambda>0: & \mathrm{d} s^{2}=\cosh ^{-2}(\sqrt{\lambda} x)\left(\mathrm{d} x^{2}+\mathrm{d} \tilde{s}^{2}\right) & (\tilde{R}>0), \\
\lambda<0: & \mathrm{d} s^{2}=\cos ^{-2}(\sqrt{-\lambda} x)\left(\mathrm{d} x^{2}+\mathrm{d} \tilde{s}^{2}\right) & (\tilde{R}<0), \\
& \mathrm{d} s^{2}=\left(-\lambda x^{2}\right)^{-1}\left(\mathrm{~d} x^{2}+\mathrm{d} \tilde{s}^{2}\right) & (\tilde{R}=0), \\
& \mathrm{d} s^{2}=\sinh ^{-2}(\sqrt{-\lambda} x)\left(\mathrm{d} x^{2}+\mathrm{d} \tilde{s}^{2}\right) & (\tilde{R}>0) .
\end{array}
$$

Note that $\tilde{R}$ and $\lambda$ are related by $|\tilde{R}|=(n-1)(n-2)|\lambda|$.

\section{Non-expanding GKS Einstein spacetimes}

Let us first consider Einstein GKS spacetimes with a non-expanding $(\theta=0)$ null KS congruence $\boldsymbol{k}$. From equation (33) it follows that the congruence is also shear-free and twist-free $\sigma=\omega=0$. Thus in this case the optical matrix vanishes

$$
L_{i j}=0
$$

and Einstein equations (30) reduce to

$$
\begin{aligned}
& \mathrm{D}^{2} \mathcal{H}=0 \\
& \delta_{i}(\mathrm{D} \mathcal{H})-2 L_{[i 1]} \mathrm{D} \mathcal{H}=0, \\
& \delta_{i}\left(\delta_{i} \mathcal{H}\right)+N_{i i} \mathrm{D} \mathcal{H}+\left(4 L_{1 i}-2 L_{i 1}+\stackrel{i}{M}_{j j}\right) \delta_{i} \mathcal{H} \\
& +2 \mathcal{H}\left(2 \delta_{i} L_{[1 i]}+4 L_{1 i} L_{[1 i]}+L_{i 1} L_{i 1}+2 L_{[1 i]} \stackrel{i}{M}_{j j}\right)+\frac{4 \mathcal{H} \Lambda}{n-1}=0 .
\end{aligned}
$$


From (46)-(48) it follows that all boost weight 0 and -1 Weyl components, as given in the Appendix A, vanish.

Proposition 4 Einstein generalized Kerr-Schild spacetimes (2) with non-expanding $K S$ congruence $\boldsymbol{k}$ are of type $N$ with $\boldsymbol{k}$ being the multiple WAND. Twist and shear of the KS congruence $\boldsymbol{k}$ necessarily vanish and these solutions thus belong to the class of Einstein type $N$ Kundt spacetimes.

Note that the above statement is also valid if we admit an additional aligned null radiation term in the Ricci tensor i.e. $R_{a b}=\frac{2 \Lambda}{n-2} g_{a b}+\Phi k_{a} k_{b}$. The aligned null radiation term appears only on the right hand side of the Einstein field equation (49) and therefore it does not affect the derivation of proposition 4 .

Kundt metrics defined as spacetimes admitting a null geodesic congruence with vanishing optical matrix $L_{i j}$ can be in $n$ dimensions expressed as [3]

$\mathrm{d} s^{2}=2 \mathrm{~d} u\left[\mathrm{~d} v+H\left(u, v, x^{k}\right) \mathrm{d} u+W_{i}\left(u, v, x^{k}\right) \mathrm{d} x^{i}\right]+g_{i j}\left(u, x^{k}\right) \mathrm{d} x^{i} \mathrm{~d} x^{j}$,

where $i, j=2 \ldots n-1$. In general Kundt spacetimes do not admit GKS form. This directly follows from the fact that there exist e.g. type III Einstein Kundt spacetimes which, by proposition 4 are incompatible with GKS form. It can be however shown [4] that all type N Ricci-flat Kundt metrics [3]

$\mathrm{d} s^{2}=2 \mathrm{~d} u\left[\mathrm{~d} v+H\left(u, v, x^{k}\right) \mathrm{d} u+W_{i}\left(u, v, x^{k}\right) \mathrm{d} x^{i}\right]+\delta_{i j} \mathrm{~d} x^{i} \mathrm{~d} x^{j}$,

where functions $W_{i}$ and $H$ are given in [3] admit KS form. Since there exist a $H^{0}\left(r, u, x^{k}\right)$ for which metric (51) is flat, all metrics (51) can be written in KerrSchild form $\mathrm{d} s^{2}=\mathrm{d} s_{\text {flat }}^{2}+\left(H^{0}-H_{\text {flat }}^{0}\right) \mathrm{d} u^{2}$.

Let us now show, using results of [16,17] (see also [18]), that all four-dimensional type N Einstein Kundt spacetimes admit GKS form. This class of metrics can be expressed as [16]

$\mathrm{d} s^{2}=-2 \frac{Q^{2}}{P^{2}} \mathrm{~d} u \mathrm{~d} v+\left(2 k \frac{Q^{2}}{P^{2}} v^{2}-\frac{\left(Q^{2}\right)_{, u}}{P^{2}} v-\frac{Q}{P} H\right) \mathrm{d} u^{2}+\frac{1}{P^{2}}\left(\mathrm{~d} x^{2}+\mathrm{d} y^{2}\right)$,

where

$$
\begin{aligned}
& P=1+\frac{\tilde{\Lambda}}{12}\left(x^{2}+y^{2}\right), \quad k=\frac{\tilde{\Lambda}}{6} \alpha(u)^{2}+\frac{1}{2}\left(\beta(u)^{2}+\gamma(u)^{2}\right), \\
& Q=\left(1-\frac{\tilde{\Lambda}}{12}\left(x^{2}+y^{2}\right)\right) \alpha(u)+\beta(u) x+\gamma(u) y,
\end{aligned}
$$

with $\tilde{\Lambda}$ being four-dimensional cosmological constant and $H=H(x, y, u)$.

These spacetimes are Einstein if

$$
P^{2}\left(H_{, x x}+H_{, y y}\right)+\frac{2}{3} \tilde{\Lambda} H=0
$$

The general solution of (54) is [17]

$$
H=2 f_{1, x}-\frac{\tilde{\Lambda}}{3 P}\left(x f_{1}+y f_{2}\right),
$$

where functions $f_{1}=f_{1}(u, x, y)$ and $f_{2}=f_{2}(u, x, y)$ are subject to $f_{1, x}=f_{2, y}$, $f_{1, y}=-f_{2, x}$. It can be shown that metrics (52) are conformally flat for

$$
H(x, y, u)=\frac{1}{P}\left(A\left(1-\frac{\tilde{\Lambda}}{12}\left(x^{2}+y^{2}\right)\right)+B x+C y\right),
$$

where $A(u), B(u)$ and $C(u)$ are arbitrary functions. Thus all metrics (52) differ from the conformally flat case only by a factor of $\mathrm{d} u$ and are therefore GKS. 


\subsection{Examples of higher dimensional GKS Einstein Kundt spacetimes}

In this section we will use Brinkmann warp product discussed in section 4 to construct examples of higher dimensional Einstein Kundt spacetimes belonging to the GKS class.

Let us first use $(44)$ to construct $(n+1)$-dimensional type N generalized KerrSchild Einstein spacetimes from $n$-dimensional vacuum type N Kundt metrics (51)

$\mathrm{d} s^{2}=\frac{1}{-\lambda \tilde{z}^{2}}\left(2 \mathrm{~d} u\left[\mathrm{~d} v+H\left(u, v, x^{k}\right) \mathrm{d} u+W_{i}\left(u, v, x^{k}\right) \mathrm{d} x^{i}\right]+\delta_{i j} \mathrm{~d} x^{i} \mathrm{~d} x^{j}+\mathrm{d} \tilde{z}^{2}\right)$,

where $i, j=2 \ldots n-1$. By performing transformation $v=-\lambda \tilde{v} \tilde{z}^{2}$ we can set the above metric to the canonical Kundt form (50)

$\mathrm{d} s^{2}=2 \mathrm{~d} u\left[\mathrm{~d} \tilde{v}+\tilde{H} \mathrm{~d} u+\tilde{W}_{\tilde{\imath}} \mathrm{d} x^{\tilde{\imath}}\right]+\frac{1}{-\lambda \tilde{z}^{2}} \delta_{\tilde{\imath} \tilde{\jmath}} \mathrm{d} x^{\tilde{\imath}} \mathrm{d} x^{\tilde{\jmath}}$

where $\tilde{\imath}, \tilde{\jmath}=2 \ldots n$.

$$
\begin{aligned}
& \tilde{H}=\frac{1}{-\lambda \tilde{z}^{2}} H\left(u, v, x^{k}\right), \\
& \tilde{W}_{i}=\frac{1}{-\lambda \tilde{z}^{2}} W_{i}\left(u, v, x^{k}\right), \quad i=1 \ldots n-2 \\
& \tilde{W}_{(n-1)}=\frac{2 \tilde{v}}{\tilde{z}} \\
& \mathrm{~d} x^{(n-1)}=\mathrm{d} \tilde{z} .
\end{aligned}
$$

Vacuum type N Kundt spacetimes are VSI (all curvature invariants, including differential invariants constructed from arbitrary covariant derivatives of Riemann tensor vanish [19]). In the case with non-vanishing cosmological constant $\Lambda$ curvature invariants either vanish or are constants depending on $\Lambda$. All non-expanding Einstein Kerr-Schild spacetimes are thus CSI (metrics with constant scalar invariants) [20]. In fact metrics (57), (58) were already discussed in [20, 21] in the context of CSI spacetimes and supergravity.

So far we used only Ricci-flat type N seed metrics. One can however also warp Einstein seed metrics (52) (Note that warping Einstein metrics (58) does not lead to new results.) In principle one can use several possible combinations of signs of Ricci scalars of the seed metric and full metric (see (42)-(45)) to construct a five-dimensional Einstein solutions from (52). Note however that only the case (43) with both Ricci scalars being negative is free from curvature or parallelly propagated singularities at $f(z)=0$ [15]. Therefore here we limit ourselves to the seed metrics (52) with $\tilde{\Lambda}<0$ and warp product (43) which leads to five dimensional metrics

$$
\begin{gathered}
\mathrm{d} s^{2}=\frac{1}{\cos ^{2}\left(\sqrt{-\frac{\tilde{\Lambda}}{3}} z\right)}\left(-2 \frac{Q^{2}}{P^{2}} \mathrm{~d} u \mathrm{~d} v+\left(2 k \frac{Q^{2}}{P^{2}} v^{2}-\frac{\left(Q^{2}\right)_{, u}}{P^{2}} v-\frac{Q}{P} H\right) \mathrm{d} u^{2}\right. \\
\left.+\frac{1}{P^{2}}\left(\mathrm{~d} x^{2}+\mathrm{d} y^{2}\right)+\mathrm{d} z^{2}\right) .
\end{gathered}
$$

The four-dimensional seed metrics - type N Kundt spacetimes with $\tilde{\Lambda}<0$ can be split to three geometrically distinct subclasses (see [18]). Depending on whether $k$ is positive, negative or vanishing, we will denote these metrics as $\operatorname{KN}\left(\tilde{\Lambda}^{-}, k^{+}\right)$, $\mathrm{KN}\left(\tilde{\Lambda}^{-}, k^{-}\right)$and $\mathrm{KN}\left(\tilde{\Lambda}^{-}, k^{0}\right)$ (generalized Siklos waves), respectively. 
$\mathrm{KN}\left(\tilde{\Lambda}^{-}, k^{+}\right)$spacetimes with the canonical choice $\alpha=0, \beta=\sqrt{2}, \gamma=0$ are represented by the metric (52) where the functions $Q$ and $k$ are given by

$$
Q=\sqrt{2} x, \quad k=1 .
$$

One may put the background AdS metric to the canonical form by performing the coordinate transformation

$$
\begin{aligned}
u & =\frac{Y \mp \sqrt{T^{2}-X^{2}-Z^{2}}}{a}, & T & =\frac{a^{2}(2-P)}{2 x v}, \\
v & = \pm \frac{a}{2 \sqrt{T^{2}-X^{2}-Z^{2}}}, & X & =\frac{a^{2} P}{2 x v}, \\
x & = \pm \frac{2 a \sqrt{T^{2}-X^{2}-Z^{2}}}{X+T}, & Y & =\frac{a(1+2 u v)}{2 v}, \\
y & =\frac{2 a Z}{X+T}, & Z & =\frac{a y}{2 x v},
\end{aligned}
$$

where $a=\sqrt{-\frac{3}{\Lambda}}$.

The case $\operatorname{KN}\left(\tilde{\Lambda}^{-}, k^{-}\right)$is represented by canonical choice $\alpha=1, \beta=0, \gamma=0$ leading to

$$
Q=1-\frac{\tilde{\Lambda}}{12}\left(x^{2}+y^{2}\right), \quad k=\frac{\tilde{\Lambda}}{6} .
$$

In this case the background AdS metric can be cast to the canonical form by using coordinate transformation

$$
\begin{array}{ll}
u=\sqrt{2}\left( \pm \sqrt{X^{2}+Y^{2}+Z^{2}}-T\right), & T=\sqrt{2} \frac{a^{2}-u v}{2 v}, \\
v= \pm \frac{a^{2}}{\sqrt{2} \sqrt{X^{2}+Y^{2}+Z^{2}}}, & X=\frac{\sqrt{2} a^{2} P}{2 Q v}, \\
x=\frac{2 a Z}{X \pm \sqrt{X^{2}+Y^{2}+Z^{2}}}, & Y=\frac{\sqrt{2} a x}{2 Q v} \\
y=\frac{2 a Y}{X \pm \sqrt{X^{2}+Y^{2}+Z^{2}}}, & Z=\frac{\sqrt{2} a y}{2 Q v},
\end{array}
$$

where $a=\sqrt{-\frac{3}{\Lambda}}$.

In the last case with seed metrics $\operatorname{KN}\left(\tilde{\Lambda}^{-}, k^{0}\right)$ the canonical choice is $\alpha=1$, $\beta=\sqrt{-\frac{1}{3} \tilde{\Lambda}} \cos \theta$ and $\gamma=\sqrt{-\frac{1}{3} \tilde{\Lambda}} \sin \theta$.

It is worth to note that in special case when $\theta$ is independent of $u$ (Siklos waves) one can obtain the same five-dimensional metric by either warping appropriate Einstein four dimensional seed metric (52) using (43) or by warping Ricci-flat $p p$ waves using (44). This is related to the fact that Siklos waves can be cast to a form conformal to pp-waves (see e.g. [18] for details).

\section{Expanding Einstein spacetimes}

\subsection{Optical constraint}

As in [4], for $\theta \neq 0$ one can express $\mathrm{D} \log \mathcal{H}$ from equation (33)

$$
\mathrm{D} \log \mathcal{H}=\frac{L_{i k} L_{i k}}{\theta(n-2)}-(n-2) \theta,
$$


which after substituting back to (31) leads to the "optical constraint" [4]

$$
L_{i k} L_{j k}=\frac{L_{l k} L_{l k}}{(n-2) \theta} S_{i j}
$$

It follows that $L_{i j}$ is also a normal matrix and thus it can be put into a block-diagonal form by appropriate spins. Furthermore, such canonical frame is compatible with parallel transport along $\boldsymbol{k}$ [22]. Consequently, dependence of the optical matrix on the affine parameter $r$ along $\boldsymbol{k}$ can be determined from Sachs equation [22, 4]. This leads to

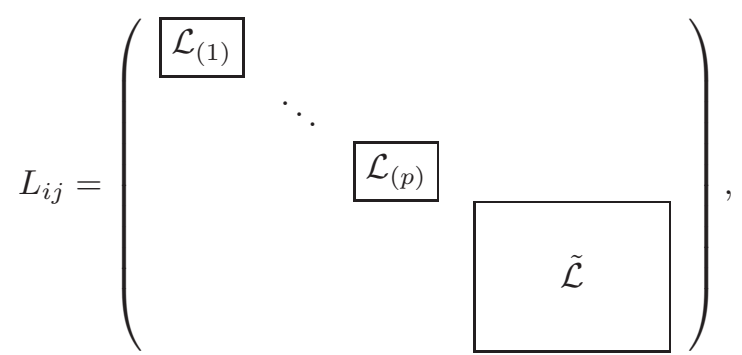

with $\mathcal{L}_{(1)}, \ldots, \mathcal{L}_{(p)}$ being $2 \times 2$ blocks of the form

$$
\begin{aligned}
& \mathcal{L}_{(\mu)}=\left(\begin{array}{cc}
s_{(2 \mu)} & A_{2 \mu, 2 \mu+1} \\
-A_{2 \mu, 2 \mu+1} & s_{(2 \mu)}
\end{array}\right) \quad(\mu=1, \ldots, p), \\
& s_{(2 \mu)}=\frac{r}{r^{2}+\left(a_{(2 \mu)}^{0}\right)^{2}}, \quad A_{2 \mu, 2 \mu+1}=\frac{a_{(2 \mu)}^{0}}{r^{2}+\left(a_{(2 \mu)}^{0}\right)^{2}},
\end{aligned}
$$

and $\tilde{\mathcal{L}}$ being $(n-2-2 p) \times(n-2-2 p)$-dimensional diagonal matrix

$$
\tilde{\mathcal{L}}=\frac{1}{r} \operatorname{diag}(\underbrace{1, \ldots, 1}_{(m-2 p)}, \underbrace{0, \ldots, 0}_{(n-2-m)})
$$

with $0 \leq 2 p \leq m \leq n-2$ and $m$ denoting the rank of $L_{i j}$.

As in [4] trace of $L_{i j}$ is

$$
(n-2) \theta=2 \sum_{\mu=1}^{p} \frac{r}{r^{2}+\left(a_{(2 \mu)}^{0}\right)^{2}}+\frac{m-2 p}{r}
$$

and

$$
L_{i k} L_{i k}=(n-2) \theta \frac{1}{r} .
$$

Using the above results we can determine the $r$-dependence of $\mathcal{H}$ by integrating (68)

$$
\mathcal{H}=\frac{\mathcal{H}_{0}}{r^{m-2 p-1}} \prod_{\mu=1}^{p} \frac{1}{r^{2}+\left(a_{(2 \mu)}^{0}\right)^{2}},
$$

which is identical to the case with vanishing $\Lambda$ discussed in detail in [4]. 


\subsection{Algebraic type}

Let us show that Weyl types III and $\mathrm{N}$ are not compatible with expanding Einstein KS spacetimes.

For types III and N, boost weight zero Weyl components vanish. In particular vanishing of $C_{0 i 1 j}$ as given in Appendix A implies

$$
L_{i j} \mathrm{DH}=2 \mathcal{H} A_{i k} L_{k j} .
$$

Multiplying the above equation with $L_{l j}$, using the optical constraint and taking the trace gives

$$
\theta \mathrm{DH}=0 .
$$

Now we can repeat the argument given in Appendix B of [4] that case DH $=0$ implies $A_{i j}=0$ and $S_{i j}=\operatorname{diag}(s, 0, \ldots, 0)$. This form of the optical matrix is not compatible with the canonical form of $L_{i j}$ for Einstein spacetimes of types III and $\mathrm{N}$ determined in [9] using Bianchi identities in the vacuum case. Since cosmological constant does not enter Bianchi identities, same results follow also for Einstein spacetimes. Note that although in the corresponding proof in [9] additional assumptions were made in the type III case, these assumptions were not used in the non-twisting case needed here. We can thus conclude that expanding Einstein GKS solutions with DH $=0$ do not exist. Then from (77)

Proposition 5 Einstein generalized Kerr-Schild spacetimes (2) with expanding KS congruence $\boldsymbol{k}$ are of Weyl types II or D or conformally flat.

\section{3. r-dependence of b.w. 0 components}

For expressing $r$-dependence of boost weight zero components of the Weyl tensor we adopt more compact notation $[12,11]$,

$$
\Phi_{i j} \equiv C_{0 i 1 j}, \quad \Phi=C_{0101}, \quad \Phi_{i j}^{S}=-\frac{1}{2} C_{i k j k}, \quad \Phi_{i j}^{A}=\frac{1}{2} C_{01 i j} .
$$

Substituting $r$-dependence of $L_{i j}$ (70)-(72) to the expressions for the corresponding Weyl tensor components given in Appendix A we immediately obtain $r$-dependence of $\Phi_{i j}$

$$
\begin{aligned}
& \Phi_{2 \mu, 2 \mu}=\Phi_{2 \mu+1,2 \mu+1}=-\mathrm{D} \mathcal{H} s_{(2 \mu)}-2 \mathcal{H} A_{2 \mu, 2 \mu+1}^{2}, \\
& \Phi_{2 \mu, 2 \mu+1}=\Phi_{2 \mu, 2 \mu+1}^{A}=-\mathrm{D}\left(\mathcal{H} A_{2 \mu, 2 \mu+1}\right), \\
& \Phi_{\alpha \beta}=-r^{-1} \delta_{\alpha \beta}, \quad \Phi=\mathrm{D}^{2} \mathcal{H} .
\end{aligned}
$$

Hence $\Phi_{i j}$ reproduces the block diagonal structure of matrix $L_{i j}$. Similarly one can determine $r$-dependence of the remaining non-vanishing boost weight zero components

$C_{2 \mu, 2 \mu+1,2 \mu, 2 \mu+1}=2 \mathcal{H}\left(3 A_{2 \mu, 2 \mu+1}^{2}-s_{(2 \mu)}^{2}\right)$,

$C_{2 \mu, 2 \mu+1,2 \nu, 2 \nu+1}=2 C_{2 \mu, 2 \nu, 2 \mu+1,2 \nu+1}=-2 C_{2 \mu, 2 \nu+1,2 \mu+1,2 \nu}=4 \mathcal{H} A_{2 \mu, 2 \mu+1} A_{2 \nu, 2 \nu+1}$,

$C_{2 \mu, 2 \nu, 2 \mu, 2 \nu}=C_{2 \mu, 2 \nu+1,2 \mu, 2 \nu+1}=-2 \mathcal{H} s_{(2 \mu)} s_{(2 \nu)}$,

$C_{(\alpha)(i)(\alpha)(i)}=-2 \mathcal{H} s_{(i)} r^{-1}$,

where $\mu \neq \nu$. 


\subsection{Singularities}

Let us briefly discuss curvature singularities of Einstein expanding GKS metrics. Since these spacetimes are by proposition 5 of types II or D (omitting the trivial conformally flat case), the Kretschmann scalar is determined by boost weight zero Weyl components

$$
\begin{aligned}
R_{a b c d} R^{a b c d} & =4\left(R_{0101}\right)^{2}-4 R_{01 i j} R_{01 i j}+8 R_{0 i 1 j} R_{0 j 1 i}+R_{i j k l} R_{i j k l} \\
& =4 \Phi^{2}+8 \Phi_{i j}^{S} \Phi_{i j}^{S}-24 \Phi_{i j}^{A} \Phi_{i j}^{A}+C_{i j k l} C_{i j k l}+\frac{8 n}{(n-1)(n-2)^{2}} \Lambda^{2} .
\end{aligned}
$$

The only additional term with respect to the vacuum case is the last constant term proportional to $\Lambda^{2}$, which clearly cannot influence singularities of the expression. Therefore, using results of [4], in the "generic" case $(2 p \neq m, 2 p \neq m-1)$ curvature singularities are located at $r=0$. Note that this case also includes all expanding, nontwisting Einstein GKS solutions, such as higher dimensional (A)dS-SchwarzschildTangherlini black holes.

In the special cases $2 p=m$ and $2 p=m-1$, presence of curvature singularity

depends on the behavior of functions $a_{(2 \mu)}^{0}$, which depend on other coordinates than $r$. If $a_{(2 \mu)}^{0}$ admit real roots at $x=x_{0}$, then a curvature singularity is located at $r=0$, $x=x_{0}$. This case corresponds e.g. to the ring shaped singularity of the Kerr-de Sitter spacetime (see Appendix B for details).

\section{Summary and discussion}

Although corresponding calculations for Einstein GKS spacetimes are considerably more involved, most of the results originally obtained in the vacuum case in [4] hold for non-vanishing cosmological constant as well.

In particular the KS vector $k$ is geodetic iff $T_{00}=T_{a b} k^{a} k^{b}$ component of the stress energy tensor vanishes. Since this holds for Einstein spacetimes, we have further assumed $\boldsymbol{k}$ being geodetic. It then can be shown that GKS spacetimes are algebraically special with $\boldsymbol{k}$ being the multiple WAND.

GKS metrics naturally split into two subclasses with expansion $\theta$ either vanishing or non-vanishing.

In the Ricci-flat case it has been shown that non-expanding KS spacetimes are equivalent to the Kundt type $\mathrm{N}$ solutions. It is not clear at present whether such equivalence holds for Einstein GKS Kundt type N as well. Here we have just shown that such equivalence holds in four dimensions and that in higher dimensions nonexpanding Einstein GKS spacetimes belong to Einstein Kundt type N. We also constructed several explicit examples of Einstein GKS Kundt spacetimes using the Brinkmann warp product.

It has been also shown that for expanding Einstein GKS spacetimes optical matrix $L_{i j}$ obeys the optical constraint. In combination with $\boldsymbol{k}$ being a WAND, it allows us to solve Sachs equation (see [22] for related discussion in more general context), determine the $r$-dependence of the optical matrix (see Appendix B for comparison of the general GKS case with the five-dimensional (A)dS-Kerr black hole), KS function $\mathcal{H}$, boost weight zero components of the Weyl tensor and Kretschmann scalar. It has been also observed that in the non-twisting case a curvature singularity is always located at $r=0$ (this for example applies to higher dimensional (A)dS-SchwarzschildTangherlini black holes), while in some twisting cases further information is needed 
(note that e.g. five-dimensional Kerr-de Sitter black hole with two non-zero spins is regular at $r=0$, while it is singular when one spin vanishes, see Appendix B for details).

In future works it would be of interest to study whether some of the above results hold in more general context, such as for Kerr-Schild spacetimes in Einstein-GaussBonnet gravity [23], for extended Kerr-Schild ansatz [24] (see also [25]) or for multiKerr-Schild form [26] and analyze what precisely are the conditions for these classes of spacetimes to admit some sort of hidden symmetries [27].

It would be also useful to employ the results of this paper for finding new expanding Einstein GKS solutions or studying possible uniqueness of higher dimensional (A)dS-Kerr black holes and related black strings/branes within this class of spacetimes.

\section{Acknowledgments}

Some calculations in this paper were performed and many others checked using computer algebra software Cadabra [28, 29]. This work has been supported by research plan No AV0Z10190503. V.P. is supported by research grant GAČR P203/10/0749, T. M. is supported by the project SVV 261301 of the Charles University in Prague.

\section{Appendix A. Riemann and Weyl components}

Riemann tensor frame components sorted by boost weight for geodetic and affinely parametrized KS vector $\boldsymbol{k}$ read

$$
\begin{aligned}
& R_{0 i 0 j}=0, \quad R_{010 i}=0, \quad R_{0 i j k}=0, \\
& R_{0101}=\mathrm{D}^{2} \mathcal{H}-\frac{2 \Lambda}{(n-2)(n-1)}, \quad R_{01 i j}=-2 A_{i j} \mathrm{D} \mathcal{H}+4 \mathcal{H} S_{k[j} A_{i] k}, \\
& R_{0 i 1 j}=-L_{i j} \mathrm{DH}+2 \mathcal{H} A_{i k} L_{k j}+\frac{2 \Lambda}{(n-2)(n-1)} \delta_{i j}, \\
& R_{i j k l}=4 \mathcal{H}\left(A_{i j} A_{k l}+A_{l[i} A_{j] k}+S_{l[i} S_{j] k}\right)+\frac{2 \Lambda}{(n-2)(n-1)}\left(\delta_{i k} \delta_{j l}-\delta_{i l} \delta_{j k}\right), \\
& R_{011 i}=-\delta_{i}(\mathrm{DH})+2 L_{[i 1]} \mathrm{D} \mathcal{H}+L_{j i} \delta_{j} \mathcal{H}+2 \mathcal{H}\left(L_{1 j} L_{j i}-L_{j 1} S_{i j}\right), \\
& R_{1 i j k}=2 L_{[j \mid i} \delta_{\mid k]} \mathcal{H}+2 A_{j k} \delta_{i} \mathcal{H}+4 \mathcal{H}\left(\delta_{[k} S_{j] i}+\stackrel{l}{M}_{[j k]} S_{i l}-\stackrel{l}{M}_{i[j} S_{k] l}\right. \\
& \left.+L_{1 i} A_{j k}+L_{1[k} A_{j] i}\right), \\
& R_{1 i 1 j}=\delta_{i}\left(\delta_{j} \mathcal{H}\right)+\stackrel{k}{M}(i j) \delta_{k} \mathcal{H}+4 L_{1(i} \delta_{j)} \mathcal{H}-2 L_{(i \mid 1} \delta_{j)} \mathcal{H}+N_{(i j)} \mathrm{D} \mathcal{H}-S_{i j} \Delta \mathcal{H} \\
& +2 \mathcal{H}\left(\delta_{(i} L_{1 \mid j)}-\Delta S_{i j}-2 L_{1(i} L_{j) 1}+2 L_{1 i} L_{1 j}-L_{k(i} N_{k \mid j)}+L_{1 k} \stackrel{k}{M}(i j)\right. \\
& \left.-2 \mathcal{H} L_{k(i} A_{j) k}-2 \mathcal{H} A_{i k} A_{j k}-L_{k(i} \stackrel{k}{M}{ }_{j) 1}-L_{(i \mid k} \stackrel{k}{M}{ }_{j) 1}\right) \text {. }
\end{aligned}
$$

Weyl frame components for GKS Einstein spaces (29) are

$$
\begin{aligned}
& C_{0 i 0 j}=0, \quad C_{010 i}=0, \quad C_{0 i j k}=0, \\
& C_{0101}=R_{0101}+\frac{2 \Lambda}{(n-2)(n-1)}, \quad C_{01 i j}=R_{01 i j},
\end{aligned}
$$


Kerr-Schild spacetimes with (A)dS background

$$
\begin{aligned}
C_{0 i 1 j} & =R_{0 i 1 j}-\frac{2 \Lambda}{(n-2)(n-1)} \delta_{i j}, \\
C_{i j k l} & =R_{i j k l}-\frac{2 \Lambda}{(n-2)(n-1)}\left(\delta_{i k} \delta_{j l}-\delta_{i l} \delta_{j k}\right), \\
C_{011 i} & =R_{011 i}, \quad C_{1 i j k}=R_{1 i j k}, \quad C_{1 i 1 j}=R_{1 i 1 j} .
\end{aligned}
$$

\section{Appendix B. Five-dimensional Kerr-(A)dS metric}

Higher dimensional Kerr-(A)dS metric in the GKS form (2) is given in [7]. In five dimensions the background metric, KS vector $\boldsymbol{k}$ and function $\mathcal{H}$ are

$$
\begin{gathered}
\overline{\mathbf{g}}=-\frac{\left(1-\lambda r^{2}\right) \Delta}{\left(1+\lambda a^{2}\right)\left(1+\lambda b^{2}\right)} \mathrm{d} t^{2}+\frac{r^{2} \rho^{2}}{\left(1-\lambda r^{2}\right)\left(r^{2}+a^{2}\right)\left(r^{2}+b^{2}\right)} \mathrm{d} r^{2}+\frac{\rho^{2}}{\Delta} \mathrm{d} \theta^{2} \\
\quad+\frac{\left(r^{2}+a^{2}\right) \sin ^{2} \theta}{1+\lambda a^{2}} \mathrm{~d} \phi^{2}+\frac{\left(r^{2}+b^{2}\right) \cos ^{2} \theta}{1+\lambda b^{2}} \mathrm{~d} \psi^{2}, \\
\boldsymbol{k}=\frac{\Delta \quad}{\left(1+\lambda a^{2}\right)\left(1+\lambda b^{2}\right)} \mathrm{d} t+\frac{r^{2} \rho^{2}}{\left(1-\lambda r^{2}\right)\left(r^{2}+a^{2}\right)\left(r^{2}+b^{2}\right)} \mathrm{d} r \\
\quad-\frac{a \sin ^{2} \theta}{1+\lambda a^{2}} \mathrm{~d} \phi-\frac{b \cos ^{2} \theta}{1+\lambda b^{2}} \mathrm{~d} \psi, \\
\mathcal{H}=-\frac{M}{\rho^{2}},
\end{gathered}
$$

where

$$
\rho^{2}=r^{2}+\nu^{2}, \quad \Delta=1+\lambda \nu^{2}, \quad \nu=\sqrt{a^{2} \cos ^{2} \theta+b^{2} \sin ^{2} \theta}
$$

and $\lambda$ is defined as in (35).

In agreement with propositions 1 and 2, the KS vector $\boldsymbol{k}$ is a geodetic multiple WAND. In fact $\boldsymbol{k}$ is also affinely parametrized. Let us complete a null frame by choosing the following null vector $\boldsymbol{n}$ and spacelike vectors $\boldsymbol{m}^{(i)}$

$$
\begin{aligned}
& \boldsymbol{k}=-\frac{1}{1-\lambda r^{2}} \frac{\partial}{\partial t}+\frac{\partial}{\partial r}-\frac{a}{r^{2}+a^{2}} \frac{\partial}{\partial \varphi}-\frac{b}{r^{2}+b^{2}} \frac{\partial}{\partial \psi}, \\
& \boldsymbol{n}=\left(\frac{1}{2} \frac{\left(1+\lambda a^{2}\right)\left(1+\lambda b^{2}\right)\left(1-\lambda r^{2}\right)}{\Delta}-\frac{M}{\rho^{2}}\right) \boldsymbol{k}+\frac{\left(1+\lambda a^{2}\right)\left(1+\lambda b^{2}\right)}{\Delta} \frac{\partial}{\partial t}, \\
& \boldsymbol{m}^{(2)}=\frac{\sqrt{\Delta}}{\rho} \frac{\partial}{\partial \theta}, \\
& \boldsymbol{m}^{(3)}=\frac{\rho \sin \theta \cos \theta}{\sqrt{\Delta} \nu}\left[\frac{\left(b^{2}-a^{2}\right)\left(1-\lambda r^{2}\right)}{\rho^{2}} \frac{\partial}{\partial r}-\frac{a\left(1+\lambda a^{2}\right)}{\left(r^{2}+a^{2}\right) \sin ^{2} \theta} \frac{\partial}{\partial \varphi}\right. \\
& \left.\quad+\frac{b\left(1+\lambda b^{2}\right)}{\left(r^{2}+b^{2}\right) \cos ^{2} \theta} \frac{\partial}{\partial \psi}\right], \\
& \boldsymbol{m}^{(4)}=\frac{a b r}{\nu}\left[\frac{1-\lambda r^{2}}{r^{2}} \frac{\partial}{\partial r}+\frac{1+\lambda a^{2}}{a\left(r^{2}+a^{2}\right)} \frac{\partial}{\partial \varphi}+\frac{1+\lambda b^{2}}{b\left(r^{2}+b^{2}\right)} \frac{\partial}{\partial \psi}\right],
\end{aligned}
$$

such that the optical matrix $L_{i j}$ takes the block-diagonal form (70). We then find a parallelly propagated frame by transforming the frame (B.3) and requiring that the block-diagonal structure of $L_{i j}$ remains unchanged. This can be achieved by performing a rotation in $\boldsymbol{m}^{(2)}, \boldsymbol{m}^{(3)}$ plane followed by a null rotation with fixed $\boldsymbol{k}$

$$
\hat{\boldsymbol{n}}=\boldsymbol{n}+z_{2} \hat{\boldsymbol{m}}^{(2)}+z_{4} \hat{\boldsymbol{m}}^{(4)}+\frac{1}{2}\left(z_{2}^{2}+z_{4}^{2}\right) \boldsymbol{k}
$$


Kerr-Schild spacetimes with (A)dS background

$$
\begin{array}{c|c}
\text { 5D Kerr-de Sitter } & \text { GKS }(n=5, m=3, p=1) \\
\hline L=\left(\begin{array}{ccc}
\frac{r}{\rho^{2}} & \frac{\nu}{\rho^{2}} & 0 \\
-\frac{\nu}{\rho^{2}} & \frac{r}{\rho^{2}} & 0 \\
0 & 0 & \frac{1}{r}
\end{array}\right) & L=\left(\begin{array}{ccc}
s_{(2)} & A_{2,3} & 0 \\
-A_{2,3} & s_{(2)} & 0 \\
0 & 0 & \frac{1}{r}
\end{array}\right) \\
\mathcal{H}=-M \frac{1}{r^{2}+\nu^{2}} & \mathcal{H}=\mathcal{H}_{0} \frac{1}{r^{2}+\left(a_{(2)}^{0}\right)^{2}} \\
s_{(2)}=\frac{r}{r^{2}+\nu^{2}} & s_{(2)}=\frac{r}{r^{2}+\left(a_{(2)}^{0}\right)^{2}} \\
A_{2,3}=\frac{\nu}{r^{2}+\nu^{2}} & A_{2,3}=\frac{a_{(2)}^{0}}{r^{2}+\left(a_{(2)}^{0}\right)^{2}}
\end{array}
$$

Table B1. Comparison of the optical matrices of five-dimensional Kerr-(Anti-)de Sitter and corresponding generalized Kerr-Schild spacetime.

$$
\begin{aligned}
\hat{\boldsymbol{m}}^{(2)} & =\frac{\nu}{\rho} \boldsymbol{m}^{(2)}-\frac{r}{\rho} \boldsymbol{m}^{(3)}-z_{2} \boldsymbol{k} \\
\hat{\boldsymbol{m}}^{(3)} & =\frac{r}{\rho} \boldsymbol{m}^{(2)}+\frac{\nu}{\rho} \boldsymbol{m}^{(3)} \\
\hat{\boldsymbol{m}}^{(4)} & =\boldsymbol{m}^{(4)}-z_{4} \boldsymbol{k}
\end{aligned}
$$

with

$$
z_{2}=-\frac{\lambda\left(a^{2}-b^{2}\right) r \sin \theta \cos \theta}{\nu}, \quad z_{4}=-\frac{\lambda a b r}{\nu} .
$$

The optical matrix $L_{i j}$ of five-dimensional Kerr-(A)dS metric is of rank $m=3$ and it contains one $2 \times 2$ block $(p=1)$. One may compare this particular $L_{i j}$ with the corresponding optical matrix (70) of general GKS spacetime (with $n=5, m=3$, $p=1$ ), see table B1. The two presented quantities are in agreement and obviously

$$
a_{(2)}^{0}=\nu, \quad \mathcal{H}_{0}=-M .
$$

Let us briefly discuss presence of curvature singularities using the results of section 6.4. If $a \neq 0, b \neq 0$ then $2 p=m-1$ and since $a_{(2)}^{0}$ does not admit roots, there are no curvature singularities in this case. If we set one of the spins to zero, e.g. $b=0$, then $a_{(2)}^{0}$ has one real root at $\theta=\frac{\pi}{2}$ corresponding to a ring shaped singularity known from the four-dimensional Kerr solution.

Putting $a=b=0$ (non-twisting case corresponding to (A)dS-SchwarzschildTangherlini limit) implies $p=0$. Since neither $2 p=m-1$ nor $2 p=m$ a curvature singularity is located at $r=0$.

\section{References}

[1] R. P. Kerr and A. Schild. Some algebraically degenerate solutions of Einstein's gravitational field equations. Proc. Symp. Appl. Math., 17:199-209, 1965.

[2] R. C. Myers and M. J. Perry. Black holes in higher dimensional space-times. Ann. Phys. (N.Y.), 172:304-347, 1986.

[3] A. Coley, A. Fuster, S. Hervik, and N. Pelavas. Higher dimensional VSI spacetimes. Class. Quantum Grav., 23:7431-7444, 2006.

[4] M. Ortaggio, V. Pravda, and A. Pravdová. Higher dimensional Kerr-Schild spacetimes. Class. Quantum Grav., 26:025008, 2009. 
[5] B. Carter. Hamilton-Jacobi and Schrodinger separable solutions of Einstein's equations. Commun. Math. Phys., 10:280, 1968.

[6] S. W. Hawking, C. J. Hunter and M. M. Taylor-Robinson. Rotation and the AdS-CFT correspondence. Phys. Rev., D59:064005, 1999.

[7] G. W. Gibbons, H. Lu, Don N. Page, and C. N. Pope. The general Kerr-de Sitter metrics in all dimensions. J. Geom. Phys., 53:49-73, 2005.

[8] T. Dereli and M. Gürses. The generalized Kerr-Schild transform in eleven-dimensional supergravity. Phys. Lett. B, 171:209-211, 1986.

[9] V. Pravda, A. Pravdová, A. Coley, and R. Milson. Bianchi identities in higher dimensions. Class. Quantum Grav., 21:2873-2897, 2004. See also V. Pravda, A. Pravdová, A. Coley and R. Milson Class. Quantum Grav. 24 (2007) 1691 (corrigendum).

[10] M. Ortaggio, V. Pravda, and A. Pravdová. Ricci identities in higher dimensions. Class. Quantum Grav., 24:1657-1664, 2007.

[11] M. Durkee, V. Pravda, A. Pravdová, and H. S. Reall. Generalization of the Geroch-Held-Penrose formalism to higher dimensions. Class. Quantum Grav., 27:215010, 2010.

[12] V. Pravda, A. Pravdová, and M. Ortaggio. Type D Einstein spacetimes in higher dimensions. Class. Quantum Grav., 24:4407-4428, 2007.

[13] N. Hamamoto, T. Houri, T. Oota, and Y. Yasui. Kerr-NUT-de Sitter curvature in all dimensions. J. Phys. A, 40:F177-F184, 2007.

[14] H. W. Brinkmann. Einstein spaces which are mapped conformally on each other. Math. Ann., 94:119-145, 1925.

[15] M. Ortaggio, V. Pravda, and A. Pravdová. On higher dimensional Einstein spacetimes with a warped extra dimension. Class. Quant. Grav., 28:105006, 2011.

[16] I. Ozsváth, I. Robinson and K. Rózga. Plane-fronted gravitational and electromagnetic waves in spaces with cosmological constant. J. Math. Phys., 26:1755-1761, 1985.

[17] J. Bičák and J. Podolský. Gravitational waves in vacuum spacetimes with cosmological constant. I. Classification and geometrical properties of nontwisting type N solutions. J. Math. Phys., 40:4495-4505, 1999.

[18] J. B. Griffiths and J. Podolský. Exact Space-Times in Einstein's General Relativity Cambridge University Press, Cambridge, 2009.

[19] A. Coley, R. Milson, V. Pravda, and A. Pravdova. Vanishing scalar invariant spacetimes in higher dimensions.. Class. Quant. Grav., 21:5519-5542, 2004.

[20] A. Coley, S. Hervik, and N. Pelavas. On spacetimes with constant scalar invariants. Class. Quant. Grav., 23:3053-3074, 2006.

[21] A. Coley, A. Fuster, and S. Hervik. Supergravity solutions with constant scalar invariants. Int. J. Mod. Phys., A24:1119-1133, 2009.

[22] M. Ortaggio, V. Pravda, and A. Pravdová. Type III and N Einstein spacetimes in higher dimensions: general properties. Phys. Rev., D82:064043, 2010.

[23] A. Anabalon et al. Kerr-Schild ansatz in Einstein-Gauss-Bonnet gravity: An exact vacuum solution in five dimensions. Class. Quant. Grav., 26:065002, 2009.

[24] A. N. Aliev and D. K. Ciftci. Note on rotating charged black holes in Einstein-Maxwell-ChernSimons theory. Phys. Rev., D79:044004, 2009.

[25] B. Ett and D. Kastor. An Extended Kerr-Schild Ansatz. Class. Quant. Grav., 27:185024, 2010.

[26] W. Chen and H. Lü. Kerr-Schild structure and harmonic 2-forms on (A)ds-Kerr-NUT metrics. Phys. Lett. B, 658:158-163, 2008.

[27] P. Krtouš, Valeri P. Frolov, and D. Kubizňák. Hidden Symmetries of Higher Dimensional Black Holes and Uniqueness of the Kerr-NUT-(A)dS spacetime. Phys. Rev., D78:064022, 2008.

[28] K. Peeters. Introducing Cadabra: A symbolic computer algebra system for field theory problems. arXiv:hep-th/0701238, 2007.

[29] K. Peeters. A field-theory motivated approach to symbolic computer algebra. Comput. Phys. Commun., 176:550, 2007. 